12th International Symposium on Cosmology and

Particle Astrophysics (CosPA 2015)

International Journal of Modern Physics: Conference Series

Vol. 43 (2016) 1660189 (9 pages)

(C) The Author(s)

DOI: $10.1142 / \mathrm{S} 2010194516601897$

\title{
The South Pole Telescope: Unraveling the Mystery of Dark Energy
}

\author{
Christian L. Reichardt \\ School of Physics, The University of Melbourne \\ Parkville VIC 3010, Australia \\ christian.reichardt@unimelb.edu.au \\ Tijmen de Haan \\ Department of Physics, University of California, Berkeley, \\ Berkeley, CA 94720 \\ tijmen@berkeley.edu \\ Lindsey E. Bleem \\ Kavli Institute for Cosmological Physics, University of Chicago, \\ 5640 South Ellis Avenue, Chicago, IL 60637 \\ bleeml@uchicago.edu \\ and the South Pole Telescope collaboration \\ Published 7 July 2016
}

The South Pole Telescope (SPT) is a 10-meter telescope designed to survey the millimeter-wave sky, taking advantage of the exceptional observing conditions at the Amundsen-Scott South Pole Station. The telescope and its ground-breaking 960-element bolometric camera finished surveying 2500 square degrees at 95.150 , and $220 \mathrm{GHz}$ in November 2011. We have discovered hundreds of galaxy clusters in the SPT-SZ survey through the Sunyaev-Zel'dovich (SZ) effect. The formation of galaxy clusters the largest bound objects in the universe is highly sensitive to dark energy and the history of structure formation. I will discuss the cosmological constraints from the SPT-SZ galaxy cluster sample as well as future prospects with the soon to-be-installed SPT-3G camera.

Keywords: Galaxies: clusters: individual, cosmology: observations

\section{Introduction}

Galaxy clusters are the most massive collapsed objects in the Universe today and form at extreme peaks in the matter density field. The abundance of galaxy clusters as a function of mass and redshift strongly depends on the cosmological model, and

This is an Open Access article published by World Scientific Publishing Company. It is distributed under the terms of the Creative Commons Attribution 4.0 (CC-BY) License. Further distribution of this work is permitted, provided the original work is properly cited. 
in particular on parameters that influence the growth of structure. By comparing the measured abundance to predictions see e.g., ${ }^{1,2,3}$, even moderate constraints on the cluster abundance can lead to strong cosmological bounds. The growth-based clusters constraints complement measurements of the power spectrum of the cosmic microwave background (CMB) and probes of geometry like baryon acoustic oscillations. Due to their largely orthogonal parameter degeneracies, the combination of growth-based and geometrical probes can yield significantly tighter constraints on the equation of state of dark energy and other parameters. Considered independently, these measurements provide test the dark energy paradigm and the validity of general relativity. ${ }^{4,5,6,7,8,9}$

The thermal Sunyaev-Zel'dovich (tSZ) effect ${ }^{10}$ refers to the inverse Compton scattering of CMB photons by hot electrons in the intra-cluster medium (ICM). The tSZ effect is the major signal from galaxy clusters at millimeter-wavelengths. The surface brightness of the tSZ effect is independent of redshift, which means that high-resolution mm-wave surveys can be used to select nearly mass-limited samples of clusters. Cluster surveys using high-resolution tSZ data are the perfect vehicle to constrain cluster abundances at high-redshifts. ${ }^{11}$

The primary limitation in galaxy clusters as cosmological tools is our imperfect knowledge of galaxy cluster masses. We can accurately predict the number of galaxy clusters as a function of mass for a model, and we can straightforwardly measure the actual cluster abundance as a function of some observable, like the tSZ decrement. The challenge is to tie the observed quantity back to the true mass. The same steep dependence of cluster abundances on mass that potentially enables powerful cosmological constraints means that misestimating the relation between the mass proxy and the true cluster mass significantly biases the resulting cosmological parameter bounds. Future optical and CMB lensing observations are expected to yield mass measurements across large cluster samples and substantially improve upon the current mass uncertainties.

In this conference, we have discussed preliminary cosmological studies using galaxy clusters from the full $2500 \mathrm{deg}^{2}$ SPT-SZ survey ${ }^{12}$, as well as expected future improvements from the SPT-3G survey that will begin in 2017. We describe the SPT-SZ and SPT-3G surveys in $\S 2$ and the SZ-selected galaxy cluster catalogs in $\S 3$. We discuss the cosmological potential of these surveys in $\S 4$ before concluding in $\S 5$.

\section{The South Pole Telescope Surveys}

The South Pole Telescope $(\mathrm{SPT})^{13}$ is located at the Amundsen-Scott South Pole station in Antarctica. The telescope was built to conduct large-area mm-wavelength surveys, with a key science driver being the discovery of galaxy clusters through the SZ effect. The 10-m diameter primary mirror yields beam sizes of $1.6^{\prime}, 1.1^{\prime}$, and $1.0^{\prime}$ at 95,150 and $220 \mathrm{GHz}$, which are well-matched to the typical angular size of a high redshift galaxy cluster. 
Table 1. The SPT Surveys

\begin{tabular}{ccccc}
\hline Name & $\begin{array}{c}\text { Area } \\
\left(\operatorname{deg}^{2}\right)\end{array}$ & $\begin{array}{c}95 \mathrm{GHz} \text { noise } \\
(\mu \mathrm{K})\end{array}$ & $\begin{array}{c}150 \mathrm{GHz} \text { noise }(\mu \mathrm{K}) \\
(\mu \mathrm{K})\end{array}$ & $\begin{array}{c}220 \mathrm{GHz} \text { noise }(\mu \mathrm{K}) \\
(\mu \mathrm{K})\end{array}$ \\
\hline SPT-SZ & 2500 & 40 & 18 & 70 \\
SPTpol & 500 & 10. & 5.4 & - \\
SPT-3G & 2500 & 4.2 & 2.5 & 4.0 \\
\hline
\end{tabular}

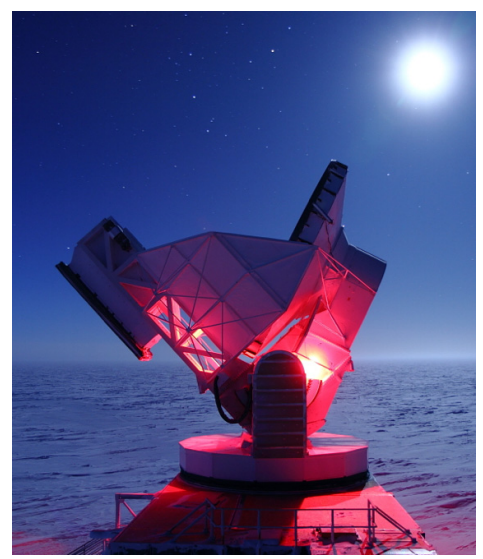

Fig. 1. The 10-m South Pole Telescope. The first camera, SPT-SZ, observed from 2007-2011. The cluster catalog in this talk is from the SPT-SZ survey. The second polarization-sensitive camera, named SPTpol, is currently observing. A third camera, SPT-3G, with ten times more detectors than SPTpol is slated to begin observations in early 2017.

Three major surveys have been conducted or are being planned with the SPT (see Table 1). This talk focused galaxy clusters discovered in the first survey, SPT$\mathrm{SZ}$, and predictions for cluster catalogs in the planned third survey, SPT-3G. The SPT-SZ survey covered $\sim 2500 \operatorname{deg}^{2}(6 \%)$ of the southern sky at 95, 150, and 220 GHz. The survey region runs from $20^{h}$ to $7^{h}$ in right ascension (R.A.) and -65 to $-40^{\circ}$ in declination (see Figure $1 \mathrm{in}^{14}$ ). The entire survey was mapped to depths of approximately 40, 18, and $70 \mu \mathrm{K}$-arcmin at 95, 150, and $220 \mathrm{GHz}$ respectively.

The planned SPT-3G survey will take advantage of a new, polarization-sensitive camera with fifteen times more detectors to observe a similar sky area $\left(\sim 2500 \mathrm{deg}^{2}\right)$ to much lower noise levels. The planned noise levels are $4.2,2.5$, and $4.0 \mu \mathrm{K}$-arcmin at 95, 150, and $220 \mathrm{GHz}$ respectively, at least seven times lower in all bands than the SPT-SZ survey. This phenomenal sensitivity will extend the resulting SZ-selected galaxy cluster catalog to much lower masses, as will be discussed in the next section. The SPT-3G survey will commence in early 2017 and run for four years.

\section{Sunyaev-Zel'dovich selected Cluster Catalogs}

The SZ effect is a superb method to locate high-redshift galaxy clusters, because the SZ surface brightness is independent of redshift. SZ surveys can potentially 


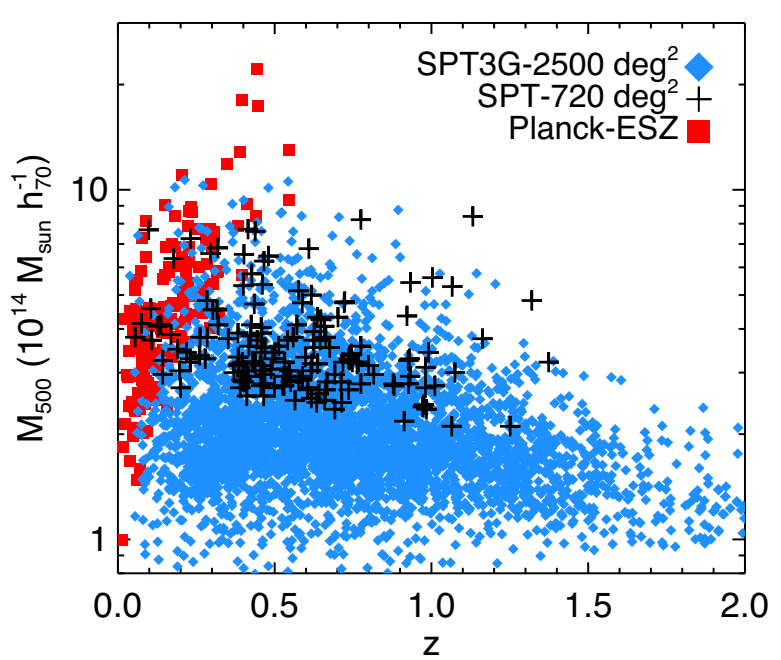

Fig. 2. SZ-selected galaxy cluster catalogs from different experiments. Red squares are clusters from the first Planck release. ${ }^{15}$ Black crosses are clusters from the first 720 deg $^{2}$ of the SPT-SZ survey, and are a representative sampling of the full survey. The smaller beam size of the SPT relative to Planck favors the detection of higher redshift clusters. Conversely, the better frequency coverage of Planck allows for better CMB subtraction and the recovery of lower-redshift galaxy clusters. Blue diamonds represent a single realization of simulated candidates for the upcoming SPT-3G survey. The SPT-3G survey will discover substantially more galaxy clusters. Figure credit: B. Benson

discover all galaxy clusters out to their redshift of formation. In this section and in Figure 2, we will briefly summarize the properties of the current SPT-SZ catalog and expected properties of the forthcoming SPT-3G survey.

\subsection{SPT-SZ cluster catalog}

The full galaxy cluster catalog from the SPT-SZ survey was recently released by Ref. 12. It contains 677 (409) galaxy cluster candidates above a detection significance of 4.5 (5) $\sigma$. Optical and NIR followup has confirmed galaxy overdensities and determined redshifts for 516 (387) of these candidates. The measured purity of $76 \%$ $(95 \%)$ is consistent with expectations from simulations. Of the confirmed clusters, $80 \%$ were first discovered in the SPT-SZ survey. The median mass of the sample is $M_{500 \mathrm{c}}\left(\rho_{\text {crit }}\right) \sim 3.5 \times 10^{14} M_{\odot} h_{70}^{-1}$, the median redshift is $z_{\text {med }}=0.55$, and the highest-redshift systems are at $z>1.4$.

\subsection{Predictions for the SPT-3G catalog}

Due to the substantially reduced noise levels expected in the SPT-3G survey (see Table 1), the mass threshold for detection is expected to be reduced by about a factor of three compared to SPT-SZ. The reduced mass threshold will lead to a much higher density of clusters on the sky: at a $4.5 \sigma$ detection significance, the 
number expected in the $2500 \mathrm{deg}^{2}$ survey increases from the current 677 candidates to a predicted 8000 candidates. This understates the improvement at a fixed purity level however, since the purity at a given significance level will be substantially better in the SPT-3G survey due to the greatly increased numbers of real clusters compared to noise fluctuations. For the chosen $4.5 \sigma$ significance, we would expect approximately 160 false detections in either catalog, which is $24 \%$ of the SPT-SZ candidates but only $2 \%$ of the SPT-3G candidates. The catalog should also extend out to higher redshifts as lower mass galaxy clusters can begin forming earlier. The highest redshift galaxy clusters in the SPT-3G sample are expected to be above a redshift of two.

\section{Cosmology with Galaxy Clusters}

As laid out in previous SPT galaxy cluster cosmology papers, ${ }^{16,17,18}$ we can lay out scaling relations between the mass, redshift, and observable quantities (namely the SPT significance and X-ray $Y_{X}$ ). Marginalizing over the scaling relation parameters encapsulates the systematic uncertainty in linking the observables to the underlying halo mass of the galaxy cluster, with priors being set on the scaling relation parameters based on previous measurements, most notably optical weak lensing measurements at low redshift that have been used to anchor the $Y_{X}$-mass relation most recently, e.g., ${ }^{19,20,21,22}$.

Applying these scaling relations to the predicted mass function for a given set of cosmological and scaling relation parameters yields a predicted number of observed clusters as a function of the observables and redshift. The predicted mass function is calculated according to the Tinker mass function.61 Recent results e.g., ${ }^{2,23}$ confirm that the Tinker mass function is more than accurate enough for the current sample size and range of cluster masses and redshifts. We compare the predicted abundance in observable space to the actual cluster sample using a binned Poisson statistic. ${ }^{24,25}$ For a given observable-space bin $x_{i}$, the probability of observing $n$ events, with expectation value $y\left(x_{i}\right)$ is

$$
P_{i}=\frac{e^{-y\left(x_{i}\right)} y\left(x_{i}\right)^{n}}{n !} .
$$

We use the Markov Chain Monte Carlo techniques to sample this space. This is implemented as a cluster likelihood module to the publicly available CosmoMC code. $^{26}$

\subsection{Cosmological implications of the SPT cluster sample}

I presented preliminary results from adding the cluster sample from the full SPT-SZ survey to other cosmological measurements of the cosmic microwave background ${ }^{27}$ Planck $+\mathrm{WP}$, Hubble constant ${ }^{28}$, baryon acoustic oscillations, ${ }^{29}$ and supernovae. ${ }^{30}$ Galaxy clusters are expected to (and do) help on parameters which significantly impact structure growth, such as the dark energy equation of state w and the sum of 
the neutrino masses. For instance when considering the dark energy equation of state $\mathrm{w}$, adding galaxy clusters to the combined dataset modestly improves constraints by a factor of 1.25 .

\subsection{Expected improvements with SPT-3G clusters}

The SPT-3G cluster survey improves upon SPT-SZ in two key ways. Most obviously, the size of the sample will grow tenfold as was noted in $§ 3$. Additionally, the low noise, polarization maps will allow cluster masses to be estimated using CMB-cluster lensing. The $\mathrm{S} / \mathrm{N}$ on individual clusters will be poor, but across the whole sample, the overall normalization can be pinned down at the $2-3 \%$ level. This is substantially better than the $\sim 15 \%$ mass calibration available today, and comparable (although somewhat lower signal-to-noise) to the mass calibration expected from optical weak lensing from LSST on the sample. The SPT-3G cluster sample should improve the DES survey's figure of merit on dark energy by a factor of four.

\section{Conclusions}

In this talk, I discussed the status of galaxy cluster surveys on the SPT. I presented the properties of the current galaxy cluster catalog from the SPT-SZ survey, as well as forecasts for a catalog in the near-future from the SPT-3 survey. Finally, I outlined how galaxy clusters could be used to constrain cosmological models and set out preliminary results on dark energy and neutrinos from these galaxy cluster catalogs. The planned SPT-3G cluster survey will lead to competitive and complementary constraints on dark energy and neutrino mass.

\section{Acknowledgments}

The South Pole Telescope program is supported by the National Science Foundation through grant ANT-0638937. Partial support is also provided by the NSF Physics Frontier Center grant PHY-0114422 to the Kavli Institute of Cosmological Physics at the University of Chicago, the Kavli Foundation, and the Gordon and Betty Moore Foundation. CR acknowledges support from the Australian Research Councils Discovery Projects scheme (DP150103208) and the University of Melbourne.

Facilities: South Pole Telescope

\section{References}

1. J. Tinker, A. V. Kravtsov, A. Klypin, K. Abazajian, M. Warren, G. Yepes, S. Gottlöber and D. E. Holz, ApJ 688, 709 (December 2008).

2. S. Bhattacharya, K. Heitmann, M. White, Z. Luki'c, C. Wagner and S. Habib, ApJ 732, p. 122 (May 2011).

3. G. P. Holder and J. E. Carlstrom, ApJ 558, 515 (September 2001).

4. M. Ishak, A. Upadhye and D. N. Spergel, Phys. Rev. D 74, p. 043513 (Aug 2006).

5. H. Zhan, L. Knox and J. A. Tyson, ApJ 690, 923 (January 2009). 
6. M. J. Mortonson, W. Hu and D. Huterer, Phys. Rev. D 79, p. 023004 (Jan 2009).

7. M. J. Mortonson, W. Hu and D. Huterer, Phys. Rev. D 81, p. 063007 (Mar 2010).

8. V. Acquaviva and E. Gawiser, Phys. Rev. D 82, p. 082001 (Oct 2010).

9. R. A. Vanderveld, M. J. Mortonson, W. Hu and T. Eifler, Phys. Rev. D 85, p. 103518 (May 2012).

10. R. A. Sunyaev and Y. B. Zel'dovich, Comments on Astrophysics and Space Physics 4, 173 (November 1972).

11. J. E. Carlstrom, G. P. Holder and E. D. Reese, ARA\&A 40, 643 (2002).

12. L. E. Bleem, B. Stalder, T. de Haan, K. A. Aird, S. W. Allen, D. E. Applegate, M. L. N. Ashby, M. Bautz, M. Bayliss, B. A. Benson, S. Bocquet, M. Brodwin, J. E. Carlstrom, C. L. Chang, I. Chiu, H. M. Cho, A. Clocchiatti, T. M. Crawford, A. T. Crites, S. Desai, J. P. Dietrich, M. A. Dobbs, R. J. Foley, W. R. Forman, E. M. George, M. D. Gladders, A. H. Gonzalez, N. W. Halverson, C. Hennig, H. Hoekstra, G. P. Holder, W. L. Holzapfel, J. D. Hrubes, C. Jones, R. Keisler, L. Knox, A. T. Lee, E. M. Leitch, J. Liu, M. Lueker, D. Luong- Van, A. Mantz, D. P. Marrone, M. McDonald, J. J. McMahon, S. S. Meyer, L. Mocanu, J. J. Mohr, S. S. Murray, S. Padin, C. Pryke, C. L. Reichardt, A. Rest, J. Ruel, J. E. Ruhl, B. R. Saliwanchik, A. Saro, J. T. Sayre, K. K. Schaffer, T. Schrabback, E. Shirokoff, J. Song, H. G. Spieler, S. A. Stanford, Z. Staniszewski, A. A. Stark, K. T. Story, C. W. Stubbs, K. Vanderlinde, J. D. Vieira, A. Vikhlinin, R. Williamson, O. Zahn and A. Zenteno, ApJS 216, p. 27 (February 2015).

13. J. E. Carlstrom, P. A. R. Ade, K. A. Aird, B. A. Benson, L. E. Bleem, S. Busetti, C. L. Chang, E. Chauvin, H.-M. Cho, T. M. Crawford, A. T. Crites, M. A. Dobbs, N. W. Halverson, S. Heimsath, W. L. Holzapfel, J. D. Hrubes, M. Joy, R. Keisler, T. M. Lanting, A. T. Lee, E. M. Leitch, J. Leong, W. Lu, M. Lueker, D. Luongvan, J. J. McMahon, J. Mehl, S. S. Meyer, J. J. Mohr, T. E. Montroy, S. Padin, T. Plagge, C. Pryke, J. E. Ruhl, K. K. Schaffer, D. Schwan, E. Shirokoff, H. G. Spieler, Z. Staniszewski, A. A. Stark, C. Tucker, K. Vanderlinde, J. D. Vieira and R. Williamson, PASP 123, 568 (May 2011).

14. K. T. Story, C. L. Reichardt, Z. Hou, R. Keisler, K. A. Aird, B. A. Benson, L. E. Bleem, J. E. Carlstrom, C. L. Chang, H.-M. Cho, T. M. Crawford, A. T. Crites, T. de Haan, M. A. Dobbs, J. Dudley, B. Follin, E. M. George, N. W. Halverson, G. P. Holder, W. L. Holzapfel, S. Hoover, J. D. Hrubes, M. Joy, L. Knox, A. T. Lee, E. M. Leitch, M. Lueker, D. Luong-Van, J. J. McMahon, J. Mehl, S. S. Meyer, M. Millea, J. J. Mohr, T. E. Montroy, S. Padin, T. Plagge, C. Pryke, J. E. Ruhl, J. T. Sayre, K. K. Schaffer, L. Shaw, E. Shirokoff, H. G. Spieler, Z. Staniszewski, A. A. Stark, A. van Engelen, K. Vanderlinde, J. D. Vieira, R. Williamson and O. Zahn, ApJ 779, p. 86 (December 2013).

15. Planck Collaboration, P. A. R. Ade, N. Aghanim, C. Armitage-Caplan, M. Arnaud, M. Ashdown, F. Atrio-Barandela, J. Aumont, H. Aussel, C. Baccigalupi and et al., $A \& A$ 571, p. A29 (November 2014).

16. K. Vanderlinde, T. M. Crawford, T. de Haan, J. P. Dudley, L. Shaw, P. A. R. Ade, K. A. Aird, B. A. Benson, L. E. Bleem, M. Brodwin, J. E. Carlstrom, C. L. Chang, A. T. Crites, S. Desai, M. A. Dobbs, R. J. Foley, E. M. George, M. D. Gladders, N. R. Hall, N. W. Halverson, F. W. High, G. P. Holder, W. L. Holzapfel, J. D. Hrubes, M. Joy, R. Keisler, L. Knox, A. T. Lee, E. M. Leitch, A. Loehr, M. Lueker, D. P. Marrone, J. J. McMahon, J. Mehl, S. S. Meyer, J. J. Mohr, T. E. Montroy, C. Ngeow, S. Padin, T. Plagge, C. Pryke, C. L. Reichardt, A. Rest, J. Ruel, J. E. Ruhl, K. K. Schaffer, E. Shirokoff, J. Song, H. G. Spieler, B. Stalder, Z. Staniszewski, A. A. Stark, C. W. Stubbs, A. van Engelen, J. D. Vieira, R. Williamson, Y. Yang, O. Zahn and A. Zenteno, ApJ 722, 1180 (October 2010). 
17. B. A. Benson, T. de Haan, J. P. Dudley, C. L. Reichardt, K. A. Aird, K. Andersson, R. Armstrong, M. L. N. Ashby, M. Bautz, M. Bayliss, G. Bazin, L. E. Bleem, M. Brodwin, J. E. Carlstrom, C. L. Chang, H. M. Cho, A. Clocchiatti, T. M. Crawford, A. T. Crites, S. Desai, M. A. Dobbs, R. J. Foley, W. R. Forman, E. M. George, M. D. Gladders, A. H. Gonzalez, N. W. Halverson, N. Harrington, F. W. High, G. P. Holder, W. L. Holzapfel, S. Hoover, J. D. Hrubes, C. Jones, M. Joy, R. Keisler, L. Knox, A. T. Lee, E. M. Leitch, J. Liu, M. Lueker, D. Luong-Van, A. Mantz, D. P. Marrone, M. McDonald, J. J. McMahon, J. Mehl, S. S. Meyer, L. Mocanu, J. J. Mohr, T. E. Montroy, S. S. Murray, T. Natoli, S. Padin, T. Plagge, C. Pryke, A. Rest, J. Ruel, J. E. Ruhl, B. R. Saliwanchik, A. Saro, J. T. Sayre, K. K. Schaffer, L. Shaw, E. Shirokoff, J. Song, H. G. Spieler, B. Stalder, Z. Staniszewski, A. A. Stark, K. Story, C. W. Stubbs, R. Suhada, A. van Engelen, K. Vanderlinde, J. D. Vieira, A. Vikhlinin, R. Williamson, O. Zahn and A. Zenteno, ApJ 763, p. 147 (February 2013).

18. C. L. Reichardt, B. Stalder, L. E. Bleem, T. E. Montroy, K. A. Aird, K. Andersson, R. Armstrong, M. L. N. Ashby, M. Bautz, M. Bayliss, G. Bazin, B. A. Benson, M. Brodwin, J. E. Carlstrom, C. L. Chang, H. M. Cho, A. Clocchiatti, T. M. Crawford, A. T. Crites, T. de Haan, S. Desai, M. A. Dobbs, J. P. Dudley, R. J. Foley, W. R. Forman, E. M. George, M. D. Gladders, A. H. Gonzalez, N. W. Halverson, N. L. Harrington, F. W. High, G. P. Holder, W. L. Holzapfel, S. Hoover, J. D. Hrubes, C. Jones, M. Joy, R. Keisler, L. Knox, A. T. Lee, E. M. Leitch, J. Liu, M. Lueker, D. Luong-Van, A. Mantz, D. P. Marrone, M. McDonald, J. J. McMahon, J. Mehl, S. S. Meyer, L. Mocanu, J. J. Mohr, S. S. Murray, T. Natoli, S. Padin, T. Plagge, C. Pryke, A. Rest, J. Ruel, J. E. Ruhl, B. R. Saliwanchik, A. Saro, J. T. Sayre, K. K. Schaffer, L. Shaw, E. Shirokoff, J. Song, H. G. Spieler, Z. Staniszewski, A. A. Stark, K. Story, C. W. Stubbs, R. Šuhada, A. van Engelen, K. Vanderlinde, J. D. Vieira, A. Vikhlinin, R. Williamson, O. Zahn and A. Zenteno, ApJ 763, p. 127 (February 2013).

19. H. Hoekstra, R. Herbonnet, A. Muzzin, A. Babul, A. Mahdavi, M. Viola and M. Cacciato, ArXiv e-prints (February 2015).

20. D. E. Applegate, A. von der Linden, P. L. Kelly, M. T. Allen, S. W. Allen, P. R. Burchat, D. L. Burke, H. Ebeling, A. Mantz and R. G. Morris, MNRAS 439, 48 (March 2014).

21. A. von der Linden, M. T. Allen, D. E. Applegate, P. L. Kelly, S. W. Allen, H. Ebeling, P. R. Burchat, D. L. Burke, D. Donovan, R. G. Morris, R. Blandford, T. Erben and A. Mantz, MNRAS 439, 2 (March 2014).

22. A. B. Mantz, A. von der Linden, S. W. Allen, D. E. Applegate, P. L. Kelly, R. G. Morris, D. A. Rapetti, R. W. Schmidt, S. Adhikari, M. T. Allen, P. R. Burchat, D. L. Burke, M. Cataneo, D. Donovan, H. Ebeling, S. Shandera and A. Wright, MNRAS 446, 2205 (January 2015).

23. S. W. Skillman, M. S. Warren, M. J. Turk, R. H. Wechsler, D. E. Holz and P. M. Sutter, ArXiv e-prints (July 2014).

24. W. Hu and A. V. Kravtsov, ApJ 584, 702 (February 2003).

25. G. Holder, ArXiv Astrophysics e-prints (February 2006).

26. A. Lewis and S. Bridle, PRD 66, 103511 (November 2002).

27. Planck Collaboration, P. A. R. Ade, N. Aghanim, C. Armitage-Caplan, M. Arnaud, M. Ashdown, F. Atrio-Barandela, J. Aumont, C. Baccigalupi, A. J. Banday and et al., A\&SA 571, p. A16 (November 2014).

28. A. G. Riess, L. Macri, S. Casertano, H. Lampeitl, H. C. Ferguson, A. V. Filippenko, S. W. Jha, W. Li and R. Chornock, ApJ 730, 119 (April 2011).

29. L. Anderson, É. Aubourg, S. Bailey, F. Beutler, V. Bhardwaj, M. Blanton, A. S. Bolton, J. Brinkmann, J. R. Brownstein, A. Burden, C.-H. Chuang, A. J. Cuesta, 
K. S. Dawson, D. J. Eisenstein, S. Escoffier, J. E. Gunn, H. Guo, S. Ho, K. Honscheid, C. Howlett, D. Kirkby, R. H. Lupton, M. Manera, C. Maraston, C. K. McBride, O. Mena, F. Montesano, R. C. Nichol, S. E. Nuza, M. D. Olmstead, N. Padmanabhan, N. Palanque-Delabrouille, J. Parejko, W. J. Percival, P. Petitjean, F. Prada, A. M. Price-Whelan, B. Reid, N. A. Roe, A. J. Ross, N. P. Ross, C. G. Sabiu, S. Saito, L. Samushia, A. G. Sánchez, D. J. Schlegel, D. P. Schneider, C. G. Scoccola, H.-J. Seo, R. A. Skibba, M. A. Strauss, M. E. C. Swanson, D. Thomas, J. L. Tinker, R. Tojeiro, M. V. Maga na, L. Verde, D. A. Wake, B. A. Weaver, D. H. Weinberg, M. White, X. $\mathrm{Xu}$, C. Yèche, I. Zehavi and G.-B. Zhao, MNRAS 441, 24 (June 2014).

30. M. Betoule, R. Kessler, J. Guy, J. Mosher, D. Hardin, R. Biswas, P. Astier, P. ElHage, M. Konig, S. Kuhlmann, J. Marriner, R. Pain, N. Regnault, C. Balland, B. A. Bassett, P. J. Brown, H. Campbell, R. G. Carlberg, F. Cellier-Holzem, D. Cinabro, A. Conley, C. B. D'Andrea, D. L. DePoy, M. Doi, R. S. Ellis, S. Fabbro, A. V. Filippenko, R. J. Foley, J. A. Frieman, D. Fouchez, L. Galbany, A. Goobar, R. R. Gupta, G. J. Hill, R. Hlozek, C. J. Hogan, I. M. Hook, D. A. Howell, S. W. Jha, L. Le Guillou, G. Leloudas, C. Lidman, J. L. Marshall, A. Möoller, A. M. Mourão, J. Neveu, R. Nichol, M. D. Olmstead, N. Palanque-Delabrouille, S. Perlmutter, J. L. Prieto, C. J. Pritchet, M. Richmond, A. G. Riess, V. Ruhlmann-Kleider, M. Sako, K. Schahmaneche, D. P. Schneider, M. Smith, J. Sollerman, M. Sullivan, N. A.Walton and C. J.Wheeler, A\&A 568, p. A22 (August 2014). 